Doug Geisler, Eva K. Grebel, and Dante Minniti, eds.

\title{
The Globular Clusters of the Fornax Dwarf Spheroidal Galaxy: Properties, Environment
}

\author{
Eduardo Hardy ${ }^{1}$ \\ National Radio Astronomy Observatory (NRAO) \\ Camino del Observatorio 1515, Santiago-Chile, ehardy@nrao.edu
}

\begin{abstract}
The power of HST to resolve dense stellar aggregates is such that most of the relevant information on the stellar populations of Fornax was obtained only recently. These results are presented here both for the globular clusters and for the field. But Fornax has probably been the most often studied dSph galaxy, partly on account of its abundant system of globular clusters. A brief retrospective is presented, not only for its historical interest but also because it reveals the striking improvement in observational tools and analysis techniques that has taken place over the last twenty years.
\end{abstract}

\section{Introduction: Why Study the Globular Clusters of Fornax?}

The Fornax dSph galaxy stands as one of the few galaxies allowing full detailed study of its stellar populations down to the oldest main sequence turn-offs, including those of its globular clusters. It probably remains the most studied galaxy of its type in the Local Group. Up until the discovery of the Sagittarius dSph (Ibata, Gilmore \& Irwin 1994) Fornax was unchallenged as the brightest known dSph and the only one with globular clusters of its own. It has five of them (Hodge 1961). A sixth suspected GC is either a chance fluctuation of the field stellar distribution (Demers, Kunkel, \& Irwin 1994), or a clustering of distant background galaxies (Stetson et al. 1998). Pending a more detailed understanding of the Sagittarius dSph, Fornax remains likely to have the highest GC frequency per unit luminosity of any known galaxy, including M87. What makes the globular clusters of Fornax highly interesting is (a) their presence in such quantities in a low luminosity, low mass galaxy; (b) their location at a distance that allows deep photometry into the main sequence and yet yields a back-to-front ratio of only about $6 \%$; (c) a significant spread in HB morphology, ideal for the study of the "second parameter" problem; (d) an apparently significant spread in cluster metallicities; (e) the possibility of comparing in detail the properties of clusters in vastly different galaxies such as Fornax and the Galaxy, a comparison that might be relevant to the understanding of the Galaxy outer halo. Notice that (b) and (d) together could help in determining the relation between the HB luminosity and the abundance, a fundamental uncertainty in

\footnotetext{
${ }^{1}$ The National Radio Astronomy Observatory is operated by Associated Universities, Inc., under a cooperative agreement with the National Science Foundation.
} 

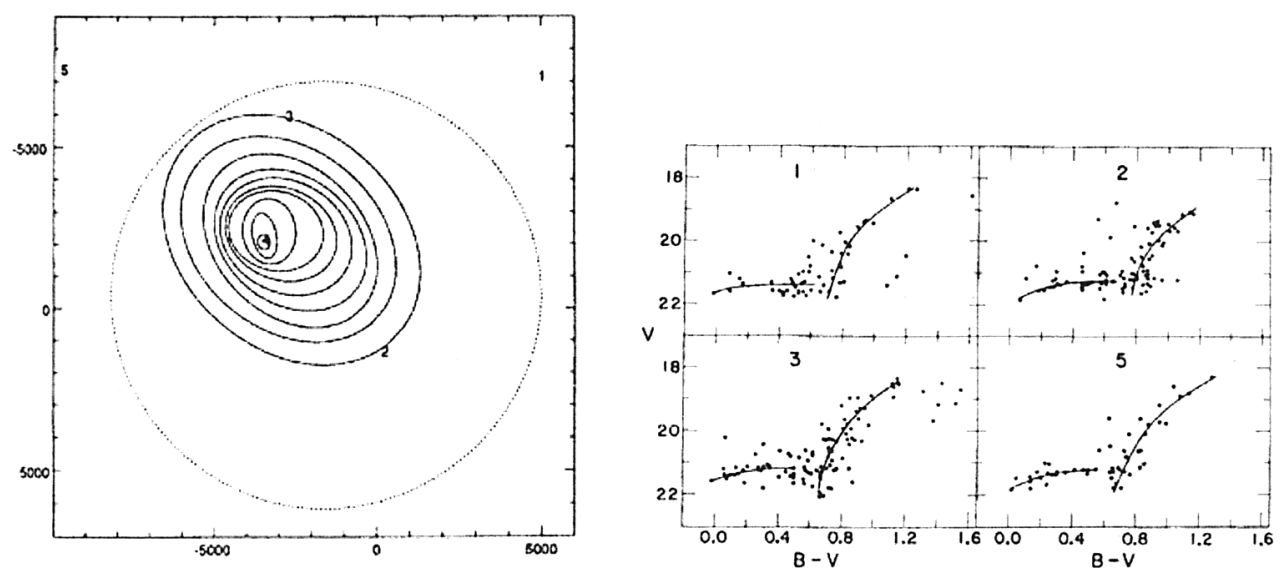

Figure 1. Left: The distribution of clusters as well as the isophotes of the field of Fornax. The box is 75.0 on a side. Right: The photographic CM diagrams of 4 clusters.

the calibration of the Pop. II distance scale. Figure 1, left, taken from Demers, Kunkel, \& Irwin (1994), shows the projected distribution of clusters over the face of Fornax. Three characteristics are noteworthy. First notice the diffuse spatial distribution of the clusters despite the fact that the clusters' orbital decay timescale is much shorter than the estimated age of the galaxy. This effect, discussed by Oh, Lin, \& Richer (2000), led them to look for a "stir up" mechanism. Secondly, notice the strong isophotal twisting and shifting, probably consistent with a Galaxy-induced tidal perturbation which in turn could also play the role of the "stir up" mechanism sought for above. Finally, notice the location of cluster 4 near the cusp of the field stellar distribution. Is Fornax a nucleated dwarf with cluster 4 as nucleus?

\section{A Retrospective: Progress Over The Last Twenty Years}

Because of the numerous investigations on the stellar populations of Fornax conducted over the years, even a short retrospective serves the purpose of showing the evolution of our understanding of this galaxy and its clusters which followed, hand in hand, the improvement in observational means and data analysis techniques. The first color-magnitude diagram study of the clusters of Fornax was conducted by Verner at al. (1981) using photographic plates obtained at the du Pont telescope. These plates, also used by Demers, Kunkel, \& Hardy (1979) for the first study of the field population of Fornax covered nearly all of the Fornax galaxy on one exposure, at the same scale of the prime focus of the Palomar $5 \mathrm{~m}$ telescope. The ensuing iris photometry did not unambiguously resolve the HB of the clusters, but provided well-defined giant branches. These giants branches demonstrated that the clusters, assuming they were old, were similar to the metal poor clusters of the Galaxy. They also provided a first determination of the distance to Fornax which has remained essentially unchanged since then. 

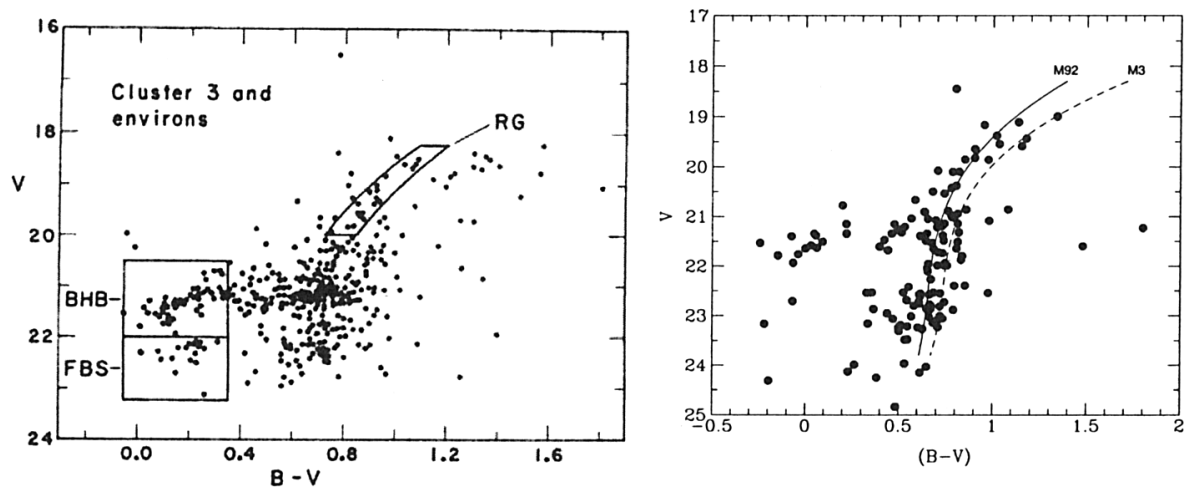

Figure 2. Left: The first CCD color-magnitude (CM) diagram of a Fornax cluster. Right: A CTIO 4m CCD CM diagram of cluster 2.

Figure 1, right, (Buonanno et al.1985) shows the CM diagrams of four of the five clusters derived from the same plate material described above but using an early version of "Romafot" in one of the first applications of PSF-fitting techniques to digitized data. The HB are well defined, although near the plate limit, and show some evidence for variations in the HB morphology from cluster to cluster. Figure 2, left, from the same paper, is the first CCD CM diagram of any Fornax GC and clearly resolves the horizontal and subgiant branches of cluster 3 . The presence in this diagram of some faint blue stars (FBS) was the first indication that a young main sequence, reaching to $\mathrm{M}_{V} \sim+1 \mathrm{mag}$, might be present in the field of Fornax, thus revealing a more complex history of star formation than hitherto assumed. The latter was consistent with the detection in the field of a wide giant branch symptomatic of a spread in the metallicities of the field stars, with the GC giant branch ridges matching the low-metallicity edge of the field distribution.

Cluster 2 was observed by Beauchamp et al. (1995) with a CCD at the PF of the CTIO $4 \mathrm{~m}$ telescope showing also for this cluster, after field subtraction, well-resolved horizontal and subgiant branches (Figure 2, right). Thus clusters 2 and 3 are low metallicity globular clusters old enough to develop an HB. But reaching the turn-off and establishing a firm age for the GCs of Fornax seemed out of the question for ground-based astronomy.

\section{Enters HST: The View From Space}

HST PC-camera observations of the GCs of Fornax (left panel of Figure 3) were published by Buonanno et al. (1998). A comparison between these observations and those on the two diagrams above shows the striking improvement in precision and depth afforded by HST. Starting with Fornax 1, the left panel of Figure 3 displays a clockwise ordering of the clusters (i.e., 1, 2, 5, 3) following increasing development of the HB towards the blue. This ordering is typified by the HB index $(B-R) /(B+V+R)$ of Lee, Demarque, \& Zinn 

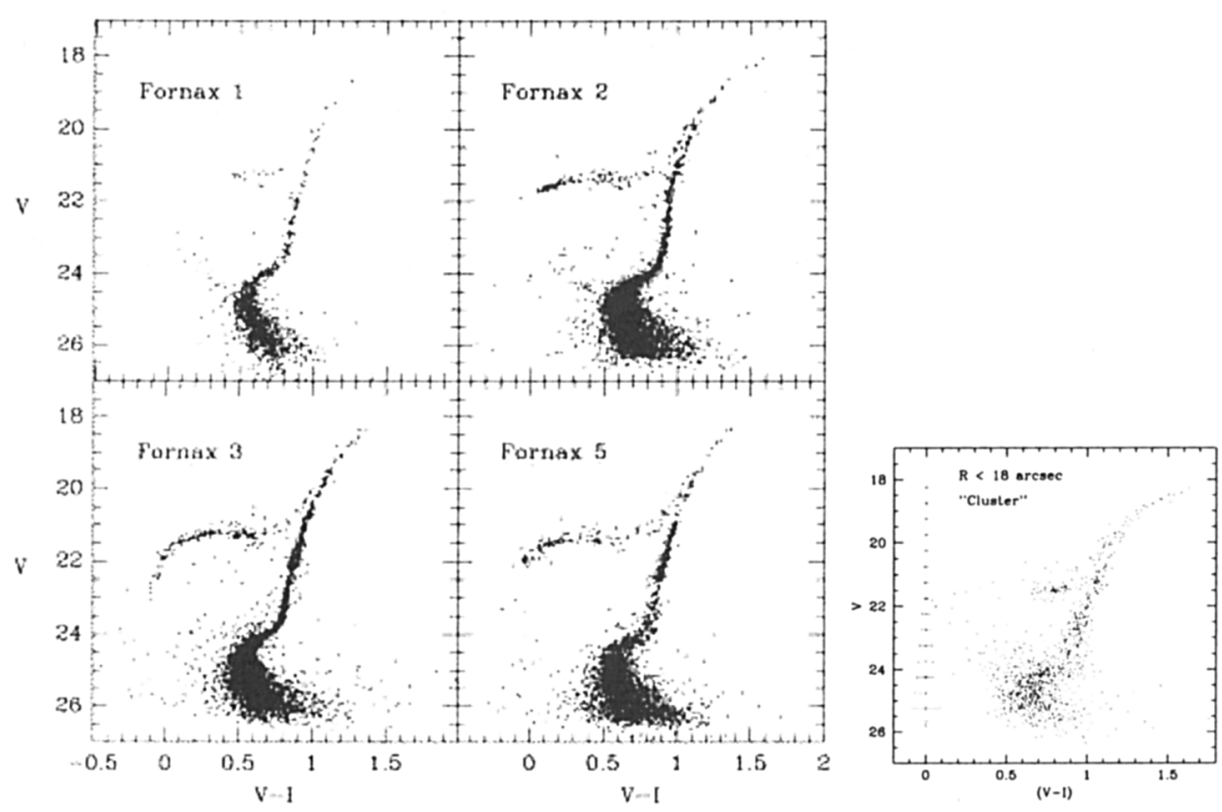

Figure 3. Left: PF HST data for clusters 1, 2, 3, 5. Right: WF HST data for cluster 4 .

(1994) which quantifies the relative number of blue (B), variable (V), and red (R) HB stars and which increases in the same order. An important result of this investigation is that the central density of the clusters $\left(\rho_{0}=M_{\odot} p c^{-3}\right)$ from Webbink (1985) also increases in the same order (see Table 1 below). Using double-differential techniques-i.e, $\Delta_{V}$, the "vertical" method, and $\delta_{(V-I)}$, the "horizontal" method-(Buonanno et al.1993; VandenBerg, Bolte, \& Stetson 1990), Buonanno et al. (1998) demonstrated that the four clusters studied have the same age within $1 \mathrm{Gyr}$, essentially the same age as the galactic GCs of similar metallicity (the average for Fornax being $\langle[\mathrm{Fe} / \mathrm{H}]\rangle=-1.98 \pm 0.17$ ). Thus, a common age marked the ignition of the old population in the Galaxy and its surroundings.

Figure 3, left, suggests that central density plays a more fundamental role than age in the "second parameter" effect, provided that the age-sensitivity of the HB (Lee, Demarque, \& Zinn 1994) had not been underestimated. It would seem that mass-loss processes related to the cluster environment are at play. The CM diagram of the elusive cluster 4, by far the more compact cluster in Fornax, was obtained by Buonanno et al. (1999) from HST Wide-Field camera archival data and is shown on the right panel of Figure 3. From integratedlight studies this cluster had been regarded as the most metal-rich (but see Beauchamp et al.1995), and thus held the promise of providing information on the HB luminosity-abundance relation. Application of the double-differential techniques revealed a significantly lower age for cluster 4 than for the rest of the Fornax system. Contrary to expectations, the properties of the giant branch on the other hand yielded an abundance $[\mathrm{Fe} / \mathrm{H}]=-1.9 \pm 0.2$ dex, essentially 
indistinguishable from that of the other clusters. Tab. 1, which reproduces Tab. 4 of Buonanno et al. (1999), contains all of the relevant up-to-date information on the cluster system of Fornax. Notice that in this table cluster 4 is the reference for the double--differential methods, and that its resulting age is $\sim 3$ Gyr younger than those of the rest. So, one of the two most extreme cases of a red-HB belongs to the cluster that is at the same time the densest and the youngest of the group. It would then seem that contrary to the previous conclusion age is indeed the "second parameter" culprit, but with central density still playing a role, perhaps when clusters age enough for their HB to start becoming blue.

Table 1: Properties of Fornax clusters

\begin{tabular}{cccccc}
\hline \hline Parameter & Cluster 1 & Cluster 2 & Cluster 3 & Cluster 4 & Cluster 5 \\
\hline$[\mathrm{Fe} / \mathrm{H}] \ldots \ldots$ & $-2.20 \pm 0.20$ & $-1.79 \pm 0.20$ & $-1.96 \pm 0.20$ & $-1.9 \pm 0.20$ & $-2.20 \pm 0.20$ \\
$\mathrm{~V}_{R} \ldots \ldots \ldots$ & $21.25 \pm 0.05$ & $21.35 \pm 0.05$ & $21.20 \pm 0.05$ & $21.52 \pm 0.05$ & $21.30 \pm 0.05$ \\
$\mathrm{E}_{V-I} \ldots \ldots \ldots$ & $0.05 \pm 0.06$ & $0.09 \pm 0.06$ & $0.05 \pm 0.06$ & $0.15 \pm 0.06$ & $0.08 \pm 0.06$ \\
$\Delta_{V} \ldots \ldots \ldots$ & $-0.25 \pm 0.15$ & $-0.22 \pm 0.15$ & $-0.31 \pm 0.15$ & - & $-0.30 \pm 0.15$ \\
$\delta_{(V-I)} \ldots \ldots$ & $0.027 \pm 0.006$ & $0.021 \pm 0.006$ & $0.035 \pm 0.006$ & - & $0.034 \pm 0.006$ \\
$\log \rho_{0} \ldots \ldots \ldots$ & 0.454 & 1.599 & 3.836 & 3.936 & 2.469 \\
$(\mathrm{~B}-\mathrm{R}) /(\mathrm{B}+\mathrm{V}+\mathrm{R})$ & $-0.2 \pm 0.2$ & $0.38 \pm 0.07$ & $0.50 \pm 0.06$ & $-1.0 \pm 0.1$ & $0.44 \pm 0.09$ \\
$\Delta t^{a} \ldots \ldots \ldots$ & $-2.6 \pm 1.7$ & $-2.5 \pm 1.7$ & $-3.2 \pm 1.7$ & - & $-3.1 \pm 1.7$ \\
$\Delta t^{b} \ldots \ldots \ldots$ & $-2.4 \pm 0.5$ & $-2.3 \pm 0.6$ & $-3.1 \pm 0.5$ & - & $-3.0 \pm 0.5$ \\
\hline
\end{tabular}

${ }^{a}$ From $\Delta_{V}$ (Gyrs)

${ }^{b}$ From $\delta_{(V-I)}$ (Gyrs)

\section{Conclusions}

The power of HST to work in extremely dense environments is such that the two publications derived from its data (Buonanno et al 1998; 1999) supersede all previous work on the clusters and contain essentially the entire history of star formation of Fornax: (a) Four of the five clusters are coeval (within $\delta_{t} \sim 1$ Gyr) with each other and with the oldest globulars of the Galaxy (i.e., M92), and have similar metallicities. (b) Cluster 4 is, however, younger than the rest by $\delta_{t} \sim 3 \mathrm{Gyr}$, although again of similar metallicity. (c) Cluster 4 is the densest and youngest and has a very red HB indicating that age determines the "second parameter" effect at least among relatively youthful globular clusters with red HB. (d) Cluster density might have an effect in the structure of the HB for the oldest clusters, perhaps by its effect on some mass-loss mechanism. (e) The study of the field surrounding cluster 4 (Buonanno et al. 1999) confirms a very long period of field star formation ( $\simeq 0.5$ to $12 \mathrm{Gyr}$ ), with the vast majority of field stars being younger than the globular clusters. (f) The precise metallicity interval of the field cannot be derived directly from the width of the giant branch, as the presence of young stars tends to mask the true interval via the age-metallicity degeneracy. Recent spectroscopic work on the near-IR Ca triplet by Pont et al. (2001) reveals a wider $[\mathrm{Fe} / \mathrm{H}]$ range than predicted from the CM diagram.

Finally, and as a global conclusion, it should be pointed out that the similarities found between the clusters of Fornax (and those of Sagittarius) and both the "normal" (e.g., M68 and M92) and "anomalous" (e.g., R106) clusters discussed in Buonanno et al. 1998; 1999), support the idea that the tidal de- 
struction of nearby dSph galaxies by the Galaxy is responsible for the diversity in properties of the GCs of the outer halo.

\section{References}

Beauchamp, D., Hardy, E., Suntzeff, N. B., \& Zinn, R. 1995, AJ, 109, 1628

Buonanno, R., Corsi, C. E., Castellani, G., Marconi, G., Fusi Pecci, F. \& Zinn, R. 1999, AJ, 118, 1671

Buonanno, R., Corsi, C. E., Zinn, R., Fusi Pecci, F., Hardy, E., \& Suntzeff, N. B. 1998, ApJ, 501, L33

Buonanno, R., Corsi, C. E., Fusi Pecci, F., Richer, H. B., \& Fahlman, G. G. 1993, AJ, 105, 184

Buonanno, R., Corsi, C. E., Fusi Pecci, F., Hardy, E., \& Zinn, R. 1985, A\&A, 152,65

Demers, S., Irwin, M. J., \& Kunkel, W. E. 1994, AJ, 108, 1648

Demers, S., Kunkel, W. E., \& Hardy, E. 1979, AJ, 232, 84

Hodge, P. W. 1961 AJ66, 83

Ibata, R. A., Gilmore, G, \& Irwin, M. J. 1994, Nature, 370. 194

Lee, Y. W., Demarque, P., \& Zinn, R. 1994, ApJ, 423, 248

Oh, K. S., Lin, D. N. C.,\& Richer, H. B. 2000 ApJ, 531, 727

Pont, F. , Gallart, C., Zinn, R., \& Hardy, E. 2001, in preparation

Stetson P.B., Hesser, J.E., \& Smecker-Hane, T.A. 1998, PASP 110, 533

VandenBerg, D. A., Bolte, M. \& Stetson. P. B. 1990, AJ, 100, 445

Verner, G., Demers, S., Hardy, E., \& Kunkel, W. E. 1981, AJ, 86, 357

Webbink. R. F. 1985, in IAU Symp. 113, Dynamics of Star Clusters, ed. J. Goodman \& P. Hut (Dordrecht: Kluwer), 541 